\title{
Application of UHPLC-ESI-QTOF-MS in Phytochemical Profiling of Sage (Salvia officinalis) and Rosemary (Rosmarinus officinalis)
}

(ㄷ) (i) $($ 우 $\odot$

\author{
Authors \\ Ravikishore Velamuri'1D, Yashaswini Sharma², John Fagan ${ }^{3}$, Jim Schaefer ${ }^{4}$
}

\author{
Affiliations \\ 1 Department of Physiology and Health, Maharishi \\ International University and Health Research Institute, \\ Fairfield, lowa \\ 2 Department of Sustainable Living, Maharishi Interna- \\ tional University, Fairfield, lowa \\ 3 Health Research Institute, Fairfield, lowa \\ 4 Soil Technologies Corp., Fairfield, lowa
}

\section{Key words}

Salvia officinalis, Rosmarinus officinalis, Lamiaceae, rosmarinic acid, ultrasound extraction, polyphenols

$$
\begin{array}{ll}
\text { received } & 10.03 .2020 \\
\text { revised } & 14.07 .2020 \\
\text { accepted } & 24.09 .2020
\end{array}
$$

\section{Bibliography}

Planta Med Int Open 2020; 7: e133-e144

DOI $10.1055 / \mathrm{a}-1272-2903$

ISSN 2509-9264

(C) 2020. The Author(s).

This is an open access article published by Thieme under the terms of the Creative Commons Attribution-NonDerivative-NonCommercial-License, permitting copying and reproduction so long as the original work is given appropriate credit. Contents may not be used for commercial purposes, or adapted remixed, transformed or built upon. (https://creativecommons.org/licenses/ by-nc-nd/4.0/)

\section{Correspondence}

Ravikishore Velamuri, PhD Scholar

Department of Physiology and Health, Maharishi International University and Health Research Institute $1000 \mathrm{~N} 4$ th Street

\section{Fairfield}

52557 lowa

USA

Tel.: +1 641552 6258, Fax: + 16414515655

rvelamuri@miu.edu

\author{
Dr. John Fagan \\ Health Research Institute \\ 505 Dimick Drive \\ Fairfield \\ 52556 lowa \\ USA \\ Tel.: +1 641552 6258, Fax: + 16414515454 \\ john.fagan@hrilabs.org
}

$\bigoplus$ Supporting information is available under http://doi. org/10.1055/a-1272-2903.

\section{ABSTRACT}

UHPLC with QTOF-MS is widely used as a powerful tool for metabolomic analysis. This technology has recently been applied to the analysis of polyphenols in food and herb extracts. Sage (Salvia officinalis) and rosemary (Rosmarinus officinalis), belonging to the family Lamiaceae, are known for their potent antioxidant properties due to the presence of polyphenols. We have developed a sensitive and reproducible UHPLC-QTOF-MS/ MS-based method for comprehensive phytochemical profiling and the identification and quantitation of specific polyphenolic compounds present in sage and rosemary leaves. The herbs were extracted ultrasonically using methanol as the solvent. In sage, rosmarinic acid $(17678.7 \pm 673.4 \mu \mathrm{g} / \mathrm{g})$ and 12-methoxy carnosic acid $(21918.3 \pm 715.4 \mu \mathrm{g} / \mathrm{g})$ were found in the highest concentrations among all polyphenols. In contrast, rosmarinic acid $(14311.0 \pm 636.4 \mu \mathrm{g} / \mathrm{g})$, luteolin-3'acetyl-O-glucuronide $(1488.50 \pm 47.58 \mu \mathrm{g} / \mathrm{g})$, and luteolin7 -O-glucuronide $(1053.68 \pm 68.83 \mu \mathrm{g} / \mathrm{g})$ were observed in the highest concentrations in rosemary. Sagerinic acid, rosmanol, rosmadial, carnosol, and carnosic acid were found in abundance in both sage and rosemary. The pentacyclic triterpenoid, corosolic acid ([M - H] $\left.]^{-} \mathrm{m} / \mathrm{z} 471.35\right)$, was detected for the first time in both plants. Of the 47 polyphenolic compounds identified in each plant, 38 compounds were found in common in rosemary and sage. A flavonoid compound, baicalin $\left([\mathrm{M}-\mathrm{H}]^{-}\right.$ $\mathrm{m} / \mathrm{z} 445.08$ ), was identified for the first time in $\mathrm{S}$. officinalis. Also, pectolinarigenin ([M - $\left.]^{-} \mathrm{m} / \mathrm{z} 313.07\right)$, a dimethoxyflavone, was detected for the first time in both sage and rosemary leaves. 


\section{Introduction}

UHPLC is an advanced technology providing improved speed of analysis while maintaining chromatographic resolution. Quadrupole time-of-flight mass spectrometry (QTOF-MS/MS) delivers rapid acquisition speed, high resolution, superior sensitivity, and excellent mass accuracy for investigating samples containing complex mixtures of compounds [1,3]. UHPLC hyphenated to atmospheric MS with its hybrid form, QTOF offers refined chromatographic peak separation and hence the most widely used tool in the profiling of polyphenolic compounds in crude samples and can identify elemental composition for both parent and fragment ions $[4,6]$. The integration of quantitative analysis and qualitative analysis is one of the important applications of this technique, which in combination with the sequential window acquisition of all theoretical fragment-ion spectra (SWATH) window of selected mass range, can be used for structural elucidation of polyphenols [7, 8]. A run time of less than $20 \mathrm{~min}$ is sufficient to carry out UHPLC analysis using smaller particle size $(<3 \mu \mathrm{M})$ analytical columns operating up to 15000 psi., which is much shorter than that under the conventional HPLC-MS method. The ESI Q-TOF detector offers excellent full-mass range detection sensitivity and a fast data acquisition rate [9].

Sage (Salvia officinalis L.) and rosemary (Rosmarinus officinalis L.), belonging to the family Lamiaceae, are the two potent aromatic and medicinal plant species used in traditional medicine, phytopharmaceutical preparations, food preservation, and aromatherapy $[10,11]$. Both herbs contain essential oils and share similar chemical and pharmacological properties. Sage is mainly used to improve cognition and is used in the treatment of cardiovascular diseases, excessive sweating, nervous disorders, depression, and cerebral ischemia $[11,14]$. It has been found to have a wide range of medicinal uses including antioxidant, antimicrobial, anti-inflammatory, anticancer, anti-dementia, hypoglycemic, and hypolipidemic effects $[15,18]$. Similarly, rosemary is traditionally used to strengthen memory functions as well as reduce headaches, tension, insomnia, fever, and respiratory system diseases $[19,20]$. It is also used as a cardiac stimulant, a strong antiseptic, antispasmodic, aromatic, carminative, emmenagogue, and nervine stimulant, and is used to cure rheumatism and dandruff [21,22]. The essential oil of both herbs is used in perfumes, as an antimicrobial, deodorant, insecticide, and fragrant repellant $[12,19]$. Sage and rosemary are rich sources of polyphenolic compounds and are the basis of widely commercialized plant extracts known for their potent antioxidant activity [14, 23].

Caffeic acid, rosmarinic acid, salvianolic acids, sagecoumarin, sagerinic acid, ferulic acid, luteolin, apigenin, hispidulin, kaempferol, and quercetin were the most abundant polyphenols present in sage [24,25], whereas in rosemary, surplus quinic acid, rosmarinic acid, gallocatechin, carnosic acid, and carnosol were reported in previous literatures [26, 27]. Earlier research has employed LC-MS methodologies to investigate the phytochemical composition of both rosemary and sage $[27,28]$. We have applied the higher resolution and discriminating power of UHPLC-QTOF-MS/MS, developing a qualitative and quantitative methodology for simultaneously identifying and quantitating the diversity of polyphenolic compounds present in sage and rosemary leaves. The method, which integrates data-independent acquisition (DAI) with the
SWATH-based fragmentation protocol and optimized UHPLC conditions, is capable of virtually exhaustive profiling of the highly complex range of phytochemicals present in both sage and rosemary. Besides this, the study also aimed at a tandem MS driven non-targeted screening of bioactive constituents by generating an accurate empirical formula aiding in orthogonal data generation. Further, the application of data evaluation platforms and compound databases can help identify compounds that lack reference standards by searching for the fragmentation spectra aiding in an untargeted screening approach.

\section{Results and Discussion}

The standard mixtures of 13 polyphenol and terpenoid compounds, apigenin, luteolin, luteolin-7-O-glucoside, hesperidin, hesperetin, rutin, narirutin, caffeic acid, chlorogenic acid, rosmarinic acid, carnosol, carnosic acid, and ursolic acid, were analyzed using the optimized UHPLC-QTOF-MS/MS method in SWATH MS² mode. The extraction window for the TOFMS mode was set at 0.02 Da for the deprotonated molecular ions. Limits of detection (LOD) for each analyte in the standard mixture was estimated using the standard deviation (STDEV) from actual values for replicate samples fortified at the limits of quantification (LOQ), applying the equation $\mathrm{LOD}=\mathrm{STDEV} \times \mathrm{t}_{0.99}\left(\mathrm{t}_{0.99}\right.$ is the $\mathrm{t}$ value at the $99 \%$ confidence level at $n-1$ degrees of freedom, where $n=6$, the number of replicates) [29]. Using the proposed UHPLC-QTOF-MS/MS method, 13 precursor polyphenols and their related characteristic compounds were successfully identified and quantified in an ultrasonic methanolic extraction of sage and rosemary. The structures of these reference standards are depicted in $>$ Fig. 1. The rosmarinic acid standard curve was used to quantify rosmarinic acid-3-O-glucoside, sagerinic acid, rosmanol, rosmadial, rosmanol methyl ether, and rosmaridiphenol. 12-Methoxy carnosic acid was quantified from the carnosic acid calibration curve, whereas micromeric acid was quantified from the ursolic acid standard curve. 6-Hydroxyluteolin-7-O-glucoside, isorhamnetin-3-O-glucoside, luteolin-7-O-glucuronide, luteolin-7-Orutinoside, scutellarin, and luteolin-3'-acetyl-O-glucuronide were all quantified by using the luteolin-7-O-glucoside standard curve, whereas apigenin-7-O-glucoside was quantified as apigenin. Concentrations of phenolic and terpenoid compounds $(\mu \mathrm{g} / \mathrm{g})$ in sage and rosemary leaf extracts analyzed through UHPLC-ESI-QTOF-MS are presented in $>$ Table $\mathbf{1}$.

The quantitative analysis of the sage extract showed that 12 -methoxy carnosic acid (21 918.33 $\pm 715.36 \mu \mathrm{g} / \mathrm{g})$ was at the highest concentration, followed by rosmarinic acid (17 678.67 \pm $673.37 \mu \mathrm{g} / \mathrm{g}$ ). Another group of abundant polyphenolic compounds was flavonoids, especially luteolin and its derivatives, phenolic diterpenoids such as rosmadial, rosmanol, and their isomers, carnosol and carnosic acid. The high content of luteolin derivatives and rosmarinic acid was also reported in previous studies on sage polyphenol analysis [30,31]. The concentration of phenolic acids, sagerinic acid, caffeic acid and its derivatives and rosmarinic acid derivatives were also high in the sage extract. Rosmarinic acid $(14311.00 \pm 636.41 \mu \mathrm{g} / \mathrm{g})$ was found in the highest concentration in rosemary, followed by luteolin 3'-acetyl-O-glucuronide ( 1488.50 $\pm 47.58 \mu \mathrm{g} / \mathrm{g})$ and luteolin-7-O-glucuronide $(1053.68 \pm 68.83 \mu \mathrm{g} / \mathrm{g})$. Hesperidin, isorhamnetin-3-O-glucoside, scutellarin, and rosmarin- 

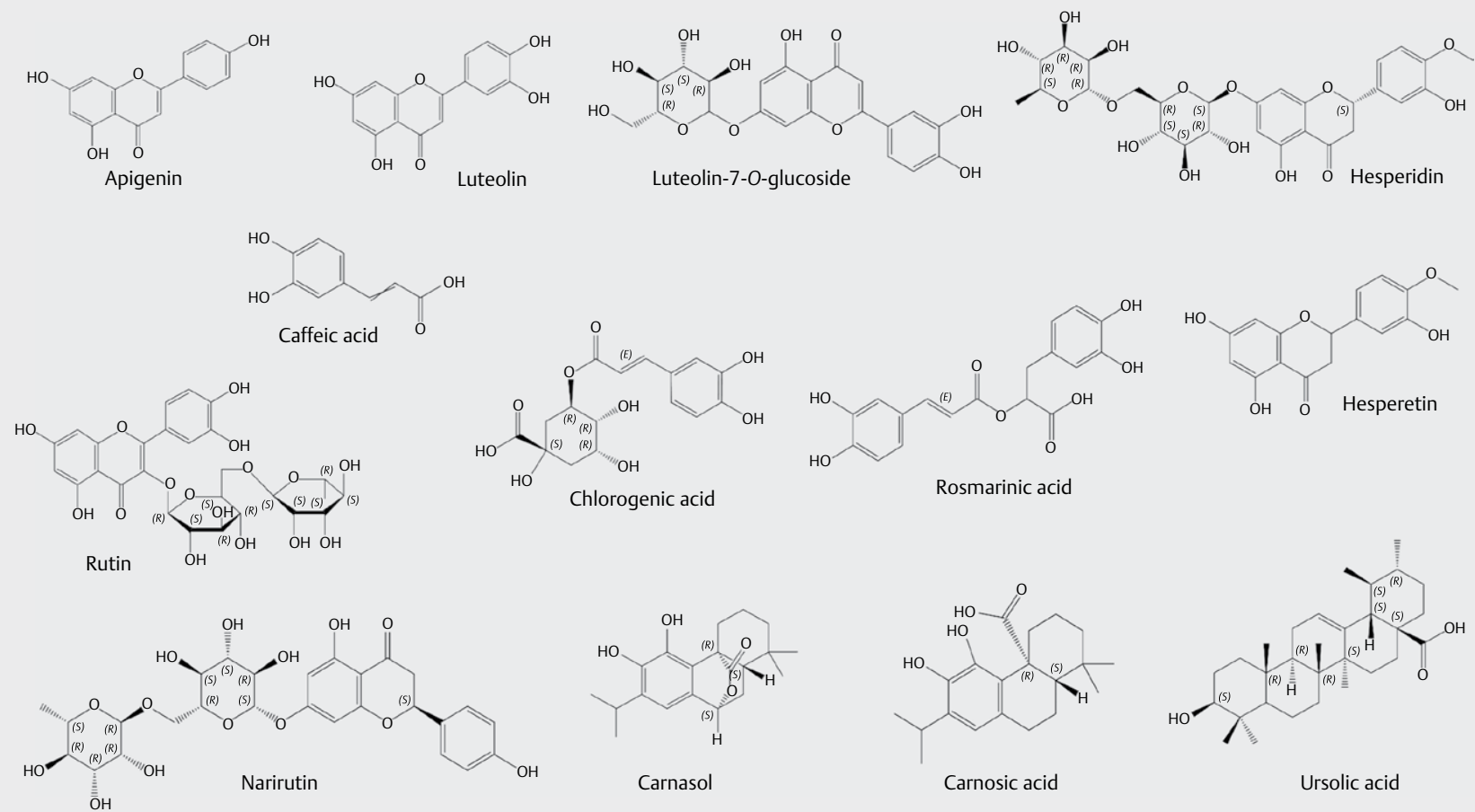

Fig. 1 Structure of phenolic compounds quantified in sage and rosemary leaves by UHPLC-QTOF-MS.

ic acid-3-O-glucoside were among the most abundant flavonoids, and the most abundant phenolic acids after rosmarinic acid were sagerinic acid and chlorogenic acid. High concentrations of phenolic diterpenoids such as rosmanol, rosmadial, and their isomers, carnosol, carnosic acid, and 12-methoxy carnosic acids were also found in both herbs. Similar results were obtained in rosemary leaves analyzed through liquid chromatography [26, 27].

Among the pentacyclic triterpenoids, micromeric acid was observed to be higher in rosemary leaves than sage, whereas the quantity of ursolic acid present in both sage and rosemary was almost equal. As compared to sage extract, rosemary extract contained higher amounts of hesperidin and rosmarinic acid-3-O-glucoside. It also contained a significant quantity of scutellarin, narirutin, and chlorogenic acid, which was absent in sage extract. Rutin and caffeic acid-3-O-glucoside were detected only in sage. Even though the chemical constituents present in sage and rosemary were similar, the concentrations of specific flavonoids, phenolic acids, and terpenoids varied significantly, showing a wide variation in their polyphenol profiles. The developed analytical method proved to be efficient, sensitive, and reproducible for the quantitative analysis of 13 compounds and their phenolic acids in the leaf extracts. All analyte peaks were well resolved within $20 \mathrm{~min}$, and the analysis simultaneously collected all of the data required for the untargeted analysis of the samples.

The high-resolution, accurate mass, UHPLC-ESI-QTOF-MS analysis used in this study not only enabled the quantitative characterization of 13 compounds for which reference materials were available, but simultaneously enabled comprehensive profiling (identification and semiquantitation) of previously unknown compounds based on their molecular formulae, exact mass measurements, and
MS/MS fragmentation patterns. Negative ionization [M - H] was reported to be more sensitive for the analysis of phenolic acids and flavonoids compared to the positive ionization mode. Hence, the analysis was carried out under the negative ionization mode $[5,32,33]$. The phenolic compounds identified in the study along with retention time, mass [M - $\mathrm{H}]^{-}$, and $\mathrm{MS}^{2}(\mathrm{~m} / \mathrm{z})$ ion fragments of sage and rosemary extracts are presented in > Tables $\mathbf{2}$ and $>\mathbf{3}$, respectively. The compounds without reference standards were identified tentatively by comparing the mass spectra data, ion fragmentation, and molecular weight $(\mathrm{m} / \mathrm{z})$ with data available in the literature and a mass spectral library obtained from the National Institutes of Standards and Technology (NIST) [26, 28, 31]. In metabolomics and especially in non-targeted metabolomic analysis, compounds are routinely identified by data processing tools that match MS/MS spectra against mass spectral reference libraries and use cheminformatics to provide spectral interpretation [34, 36]. SWATH is a DIA in LCMS/MS and provides a more comprehensive untargeted acquisition of molecular data. We used DIA methods to obtain all fragment ions for all precursors simultaneously, thereby increasing the coverage of observable molecules and reducing false negative identifications. The SWATH acquisition covered a larger number of polyphenolic compounds in the negative ionization mode.

The chromatograms of the UHPLC-ESI-QTOF-MS analysis of sage and rosemary extracts are presented in $>$ Figs. 2 and $>\mathbf{3}$, respectively. A total number of 47 phenolic and terpenoid compounds were tentatively identified in sage and rosemary leaf extracts ( $\triangleright$ Tables 2 and $\$ 3$ ). About 22 flavonoid compounds were detected in both samples, mainly representing luteolin, isorhamnetin, hispidulin, hesperidin, apigenin, and their derivatives, scutellarin, 
- Table 1 Polyphenol and terpenoid compounds concentration $(\mu \mathrm{g} / \mathrm{g})$ in sage and rosemary analyzed by UHPLC-QTOF-MS.

\begin{tabular}{|c|c|c|c|c|}
\hline & \multirow[t]{2}{*}{ Phenolic and terpenoid compounds } & \multicolumn{2}{|c|}{ Concentration $(\mu \mathrm{g} / \mathrm{g})$} & \multirow[t]{2}{*}{ Quantified as } \\
\hline & & Sage & Rosemary & \\
\hline 1 & Apigenin & $24.80 \pm 1.31$ & $6.90 \pm 0.59$ & \\
\hline 2 & Apigenin-7-O-glucoside & $125.94 \pm 9.80$ & $14.48 \pm 0.94$ & Apigenin \\
\hline 3 & Luteolin & $10.93 \pm 1.24$ & $11.13 \pm 1.25$ & \\
\hline 4 & Luteolin-7-O-glucoside & $294.33 \pm 19.44$ & $70.87 \pm 7.20$ & \\
\hline 5 & 6-Hydroxyluteolin-7-O-glucoside & $29.01 \pm 1.27$ & $49.03 \pm 5.07$ & Luteolin-7-glucoside \\
\hline 6 & Isorhamnetin-3-O-glucoside & $75.26 \pm 3.51$ & $416.37 \pm 17.99$ & Luteolin-7-glucoside \\
\hline 7 & Luteolin 7-O-glucuronide & $747.64 \pm 30.31$ & $1053.68 \pm 68.83$ & Luteolin-7-glucoside \\
\hline 8 & Luteolin-7-O-rutinoside & $153.04 \pm 6.29$ & - & Luteolin-7-glucoside \\
\hline 9 & Scutellarin & - & $152.60 \pm 11.87$ & Luteolin-7-glucoside \\
\hline 10 & Luteolin 3'-acetyl-O-glucuronide & - & $1488.50 \pm 47.58$ & Luteolin-7-glucoside \\
\hline 11 & Rutin & $10.54 \pm 1.26$ & - & \\
\hline 12 & Narirutin & - & $1.98 \pm 0.14$ & \\
\hline 13 & Hesperidin & $7.67 \pm 1.03$ & $729.22 \pm 39.36$ & \\
\hline 14 & Hesperetin & $1.99 \pm 0.26$ & $0.40 \pm 0.08$ & \\
\hline 15 & Rosmarinic acid-3-O-glucoside & $29.65 \pm 1.64$ & $331.36 \pm 14.42$ & Rosmarinic acid \\
\hline 16 & Rosmarinic acid-3-O-glucoside isomer & $20.43 \pm 2.02$ & - & Rosmarinic acid \\
\hline 17 & Caffeic acid-3-O-glucoside & $558.83 \pm 42.26$ & - & Caffeic acid \\
\hline 18 & Caffeic acid & $164.85 \pm 18.59$ & $39.08 \pm 2.73$ & \\
\hline 19 & Chlorogenic acid & - & $241.07 \pm 10.82$ & \\
\hline 20 & Rosmarinic acid & $17678.67 \pm 673.37$ & $14311.00 \pm 636.41$ & \\
\hline 21 & Sagerinic acid & $867.40 \pm 43.79$ & $819.93 \pm 46.07$ & Rosmarinic acid \\
\hline 22 & Epirosmanol & $200.16 \pm 6.31$ & $225.94 \pm 4.49$ & Rosmarinic acid \\
\hline 23 & Rosmanol & $87.42 \pm 2.97$ & $218.48 \pm 11.70$ & Rosmarinic acid \\
\hline 24 & Epiisorosmanol & $69.55 \pm 4.39$ & $182.98 \pm 7.36$ & Rosmarinic acid \\
\hline 25 & Rosmanol isomer & $29.20 \pm 1.01$ & - & Rosmarinic acid \\
\hline 26 & Rosmadial isomer-I & $268.42 \pm 20.91$ & $422.03 \pm 19.93$ & Rosmarinic acid \\
\hline 27 & Rosmadial & $342.85 \pm 6.64$ & $588.64 \pm 24.14$ & Rosmarinic acid \\
\hline 28 & Rosmadial isomer-II & $371.97 \pm 17.21$ & $358.32 \pm 26.60$ & Rosmarinic acid \\
\hline 29 & Rosmadial isomer-III & $69.33 \pm 3.23$ & - & Rosmarinic acid \\
\hline 30 & Rosmanol methyl ether & $47.12 \pm 3.08$ & $114.15 \pm 13.27$ & Rosmarinic acid \\
\hline 31 & Rosmanol methyl ether isomer & $122.13 \pm 7.25$ & $42.37 \pm 1.46$ & Rosmarinic acid \\
\hline 32 & Rosmaridiphenol & $25.57 \pm 0.94$ & - & Rosmarinic acid \\
\hline 33 & Carnosol & $544.97 \pm 33.23$ & $698.78 \pm 21.07$ & \\
\hline 34 & Carnosic acid & $555.23 \pm 23.68$ & $797.75 \pm 32.70$ & \\
\hline 35 & 12-Methoxy carnosic acid & $21918.33 \pm 715.36$ & $982.78 \pm 32.77$ & Carnosic acid \\
\hline 36 & Ursolic acid & $61.46 \pm 2.48$ & $59.03 \pm 3.95$ & \\
\hline 37 & Micromeric acid & $4.80 \pm 0.14$ & $83.22 \pm 2.38$ & Ursolic acid \\
\hline
\end{tabular}

rutin, baicalin, pectolinarigenin, and genkwanin. In sage, a dihydroxyflavone, baicalin ([M - H] $]^{-} \mathrm{m} / \mathrm{z}$ 445.08), was detected for the first time, and the flavonoid was not identified in $S$. officinalis before. Baicalin is a flavone glycoside commonly found in roots of Scutellaria baicalensis, and has been reported as having anti-inflammatory, antiviral, anti-allergic, cognitive, and neuroprotective properties [37,38]. A trihydroxy-methoxyflavone, diosmetin ([M $-\mathrm{H}]^{-} \mathrm{m} / \mathrm{z}$ 299.05), detected in the rosemary sample was previously described by Borras-Linares et al. with a similar fragmentation pattern [39]. Pectolinarigenin ([M - H] $]^{-} \mathrm{m} / \mathrm{z}$ 313.07) was yet another flavone reported for the first time in both sage and rosemary.
Pectolinarigenin was found in Salvia hypoleuca and Salvia pedicellata species, and it was also reported before in rosemary as a dimethoxyflavone with the same fragment ions [24,27]. Both diosmetin and pectolinarigenin flavonoids are said to have potent anti-inflammatory and anticancer properties [40,41]. Among the nine different identified phenolic acids, ferulic acid $\left([\mathrm{M}-\mathrm{H}]^{-}\right.$at $\mathrm{m} / \mathrm{z}$ 193.95), rosmarinic acid $\left([\mathrm{M}-\mathrm{H}]^{-}\right.$at $\left.\mathrm{m} / \mathrm{z} 359.08\right)$, salvianolic acid $\mathrm{K}\left([\mathrm{M}-\mathrm{H}]^{-}\right.$ at $m / z$ 555.12), and methyl rosmarinate ([M - H] $]^{-}$at $m / z$ 373.09) shared many of the same MS/MS $(\mathrm{m} / \mathrm{z})$ ion fragments, which are related to caffeic acid. Many phenolic acids of the Salvia species were previously reported to be caffeic acid derivatives, mostly 
> Table 2 Polyphenolic and terpenoid compounds identified by UHPLC-ESI- QTOF-MS analysis in sage (S. officinalis).

\begin{tabular}{|c|c|c|c|c|c|}
\hline SI No & Compound & RT (min) & Mass $[M-H]^{-}(m / z)$ & Formula & $M^{2}(m / z)$ Fragments \\
\hline 1 & Caffeic acid 3-O-glucoside & 0.23 & 341.09 & $\mathrm{C}_{15} \mathrm{H}_{18} \mathrm{O}_{9}$ & $179.0551(67)$ \\
\hline 2 & Caffeic acid & 0.61 & 179.04 & $\mathrm{C}_{9} \mathrm{H}_{8} \mathrm{O}_{4}$ & $135.0432(100), 134.0354(28), 105.0335(5)$ \\
\hline 3 & Gallocatechin & 0.74 & 305.07 & $\mathrm{C}_{15} \mathrm{H}_{14} \mathrm{O}_{7}$ & 225.1112 (73), 96.9579 (38) \\
\hline 4 & Luteolin-7-O-rutinoside & 0.95 & 593.12 & $\mathrm{C}_{27} \mathrm{H}_{30} \mathrm{O}_{15}$ & 287.0548 (62), $285.0386(45)$ \\
\hline 5 & Vanillic acid & 1.01 & 167.03 & $\mathrm{C}_{8} \mathrm{H}_{8} \mathrm{O}_{4}$ & $108.0208(19), 65.0388(18)$ \\
\hline 6 & Ferulic acid & 1.35 & 193.05 & $\mathrm{C}_{10} \mathrm{H}_{10} \mathrm{O}_{4}$ & 134.0366 (100), 179.0269 (41), $133.0292(18)$ \\
\hline 7 & 6-Hydroxyluteolin -7-O-glucuronide & 1.44 & 477.07 & $\mathrm{C}_{21} \mathrm{H}_{18} \mathrm{O}_{13}$ & $301.0345(100)$ \\
\hline 8 & 6-Hydroxyluteolin-7-O-glucoside & 1.57 & 463.09 & $\mathrm{C}_{21} \mathrm{H}_{20} \mathrm{O}_{12}$ & 287.0576 (25), $301.0338(10)$ \\
\hline 9 & Scutellarin & 1.76 & 461.07 & $\mathrm{C}_{21} \mathrm{H}_{18} \mathrm{O}_{12}$ & $285.0385(100)$ \\
\hline 10 & Rutin & 1.84 & 609.15 & $\mathrm{C}_{27} \mathrm{H}_{30} \mathrm{O}_{16}$ & $300.0350(10)$ \\
\hline 11 & Luteolin-7-O-glucoside & 1.92 & 447.09 & $\mathrm{C}_{21} \mathrm{H}_{20} \mathrm{O}_{11}$ & $285.0403(100)$ \\
\hline 12 & Isorhamnetin-3-O-glucoside & 1.94 & 477.10 & $\mathrm{C}_{22} \mathrm{H}_{22} \mathrm{O}_{12}$ & $315.0694(24)$ \\
\hline 13 & Baicalin & 2.03 & 445.08 & $\mathrm{C}_{21} \mathrm{H}_{18} \mathrm{O}_{11}$ & $269.0445(100)$ \\
\hline 14 & Sagerinic acid & 2.10 & 719.16 & $\mathrm{C}_{36} \mathrm{H}_{32} \mathrm{O}_{16}$ & $359.0724(100)$ \\
\hline 15 & Rosmarinic acid & 2.13 & 359.08 & $\mathrm{C}_{18} \mathrm{H}_{16} \mathrm{O}_{8}$ & 161.0216 (100), 179.0325 (59), 197.0432 (31) \\
\hline 16 & Salvianolic acid $\mathrm{K}$ & 2.30 & 555.12 & $\mathrm{C}_{27} \mathrm{H}_{24} \mathrm{O}_{13}$ & $\begin{array}{c}359.0734(100), 493.1082(44), 179.0323(16), \\
135.0426(12)\end{array}$ \\
\hline 17 & Apigenin-7-O-glucoside & 2.32 & 431.10 & $\mathrm{C}_{21} \mathrm{H}_{20} \mathrm{O}_{10}$ & $269.0825(17)$ \\
\hline 18 & Hispidulin glucuronide & 2.34 & 475.09 & $\mathrm{C}_{22} \mathrm{H}_{20} \mathrm{O}_{12}$ & 299.0552 (100), $284.0320(10)$ \\
\hline 19 & Hesperidin & 2.65 & 609.18 & $\mathrm{C}_{28} \mathrm{H}_{34} \mathrm{O}_{15}$ & $301.0697(100)$ \\
\hline 20 & 4,5,7-Trihydroxy flavone & 2.70 & 269.05 & $\mathrm{C}_{15} \mathrm{H}_{10} \mathrm{O}_{5}$ & 225.1684 (3), 201.0567 (2), 183.0452 (2) \\
\hline 21 & Methyl rosmarinate & 2.70 & 373.09 & $\mathrm{C}_{19} \mathrm{H}_{18} \mathrm{O}_{8}$ & $179.0343(100), 197.0453(15), 135.0443(9)$ \\
\hline 22 & Luteolin & 2.88 & 285.04 & $\mathrm{C}_{15} \mathrm{H}_{10} \mathrm{O}_{6}$ & $\begin{array}{c}133.0300 \text { (19), } 151.0042(12), 175.0409(9), \\
199.0406(9)\end{array}$ \\
\hline 23 & Isorhamnetin & 2.99 & 315.05 & $\mathrm{C}_{16} \mathrm{H}_{12} \mathrm{O}_{7}$ & $300.0268(100)$ \\
\hline 24 & Salvianolic acid B & 3.23 & 717.14 & $\mathrm{C}_{36} \mathrm{H}_{30} \mathrm{O}_{16}$ & 519.0935 (100), $339.0511(17)$ \\
\hline 25 & 4,5,7-Trihydroxy flavone isomer & 3.26 & 269.05 & $\mathrm{C}_{15} \mathrm{H}_{10} \mathrm{O}_{5}$ & 225.0570 (8), 159.0454 (4), 201.0568 (3) \\
\hline 26 & Hispidulin & 3.36 & 299.06 & $\mathrm{C}_{16} \mathrm{H}_{12} \mathrm{O}_{6}$ & $\begin{aligned} & 284.0315(100), 136.9866(18), 200.0475(9) \\
& 65.0019(4)\end{aligned}$ \\
\hline 27 & Hesperetin & 3.48 & 301.07 & $\mathrm{C}_{16} \mathrm{H}_{14} \mathrm{O}_{6}$ & $284.0319(100), 136.9878(20), 164.0113(5)$ \\
\hline 28 & Apigenin & 3.50 & 269.05 & $\mathrm{C}_{15} \mathrm{H}_{10} \mathrm{O}_{5}$ & 117.0346 (19), 149.0244 (14), 107.0143 (4) \\
\hline 29 & Epirosmanol & 3.82 & 345.17 & $\mathrm{C}_{20} \mathrm{H}_{26} \mathrm{O}_{5}$ & $284.1679(12), 283.8697(4)$ \\
\hline 30 & Pectolinarigenin & 3.94 & 313.07 & $\mathrm{C}_{17} \mathrm{H}_{14} \mathrm{O}_{6}$ & $\begin{array}{c}284.0266 \text { (100), } 298.0467 \text { (100), } 227.0346(13), \\
117.0337 \text { (7), } 163.0035 \text { (10) }\end{array}$ \\
\hline 31 & Rosmanol & 4.04 & 345.17 & $\mathrm{C}_{20} \mathrm{H}_{26} \mathrm{O}_{5}$ & 301.1786 (100), $284.1701(22)$ \\
\hline 32 & Rosmadial isomer & 4.31 & 343.15 & $\mathrm{C}_{20} \mathrm{H}_{24} \mathrm{O}_{5}$ & $284.1410(39)$ \\
\hline 33 & Epiisorosmanol & 4.36 & 345.17 & $\mathrm{C}_{20} \mathrm{H}_{26} \mathrm{O}_{5}$ & 284.1680 (15), 283.8695 (5) \\
\hline 34 & Genkwanin & 4.38 & 283.06 & $\mathrm{C}_{16} \mathrm{H}_{12} \mathrm{O}_{5}$ & $268.0382(85), 117.0343(10), 240.0438(12)$ \\
\hline 35 & Rosmanol isomer & 4.87 & 345.17 & $\mathrm{C}_{20} \mathrm{H}_{26} \mathrm{O}_{5}$ & $301.1786(100)$ \\
\hline 36 & Asiatic acid & 5.17 & 487.34 & $\mathrm{C}_{30} \mathrm{H}_{48} \mathrm{O}_{5}$ & - \\
\hline 37 & Rosmadial & 5.32 & 343.15 & $\mathrm{C}_{20} \mathrm{H}_{24} \mathrm{O}_{5}$ & 300.1660 (100), $284.1410(39)$ \\
\hline 38 & Rosmanol methyl ether & 5.47 & 359.99 & $\mathrm{C}_{21} \mathrm{H}_{28} \mathrm{O}_{5}$ & $\begin{array}{c}283.1657 \text { (100), } 284.1679 \text { (79), } 268.1440(20), \\
329.1699(12)\end{array}$ \\
\hline 39 & Carnosol & 5.58 & 329.18 & $\mathrm{C}_{20} \mathrm{H}_{26} \mathrm{O}_{4}$ & $\begin{array}{c}286.1847 \text { (100), } 285.05858891 \text { (100), } 270.1605(8), \\
201.0885(5)\end{array}$ \\
\hline 40 & Rosmadial isomer & 5.85 & 343.19 & $\mathrm{C}_{20} \mathrm{H}_{24} \mathrm{O}_{5}$ & 299.2016 (100), 284.1791 (45), 269.1556 (9) \\
\hline 41 & Rosmaridiphenol & 5.97 & 315.20 & $\mathrm{C}_{20} \mathrm{H}_{28} \mathrm{O}_{3}$ & $284.1768(34)$ \\
\hline 42 & Carnosic acid & 6.38 & 331.19 & $\mathrm{C}_{20} \mathrm{H}_{28} \mathrm{O}_{4}$ & 288.2043 (100), 287.2015 (39), 244.1476 (19) \\
\hline 43 & Corosolic acid & 6.44 & 471.35 & $\mathrm{C}_{30} \mathrm{H}_{48} \mathrm{O}_{4}$ & - \\
\hline 44 & 12-Methoxy-carnosic acid & 6.82 & 345.21 & $\mathrm{C}_{21} \mathrm{H}_{30} \mathrm{O}_{4}$ & 301.2138 (100), 287.1911 (89) \\
\hline 45 & Micromeric acid & 7.51 & 453.34 & $\mathrm{C}_{30} \mathrm{H}_{46} \mathrm{O}_{3}$ & - \\
\hline 46 & Betulinic acid & 7.71 & 455.35 & $\mathrm{C}_{30} \mathrm{H}_{48} \mathrm{O}_{3}$ & - \\
\hline 47 & Ursolic acid & 7.91 & 455.35 & $\mathrm{C}_{30} \mathrm{H}_{48} \mathrm{O}_{3}$ & - \\
\hline
\end{tabular}


- Table 3 Polyphenolic and terpenoid compounds identified by UHPLC-ESI-QTOF-MS analysis in rosemary (R. officinalis).

\begin{tabular}{|c|c|c|c|c|c|}
\hline SI No & Compound & RT (min) & $\begin{array}{c}\text { Mass }[\mathrm{M}-\mathrm{H}]^{-} \\
(\mathrm{m} / \mathrm{z})\end{array}$ & Formula & $\mathrm{MS}^{2}(\mathrm{~m} / \mathrm{z})$ Fragments \\
\hline 1 & Caffeoyl-fructosyl-glucose & 0.24 & 503.13 & $\mathrm{C}_{21} \mathrm{H}_{28} \mathrm{O}_{14}$ & $191.0543(81), 145.0618(72), 179.0549(63)$ \\
\hline 2 & Quinic acid & 0.43 & 191.06 & $\mathrm{C}_{7} \mathrm{H}_{12} \mathrm{O}_{6}$ & 85.0293 (45), 127.0396 (31), 93.0345 (30) \\
\hline 3 & Chlorogenic acid & 0.48 & 353.09 & $\mathrm{C}_{16} \mathrm{H}_{18} \mathrm{O}_{9}$ & $191.0534(100), 161.0239(6)$ \\
\hline 4 & Gallocatechin & 0.58 & 305.07 & $\mathrm{C}_{15} \mathrm{H}_{14} \mathrm{O}_{7}$ & 225.1078 (43), 96.9559 (33), 135.0793 (31) \\
\hline 5 & Vanillic acid & 0.67 & 167.03 & $\mathrm{C}_{8} \mathrm{H}_{8} \mathrm{O}_{4}$ & 152.0110 (60), 108.0210 (35), 123.0440 (10), 91.0195 (5) \\
\hline 6 & Caffeic acid & 0.85 & 179.03 & $\mathrm{C}_{9} \mathrm{H}_{8} \mathrm{O}_{4}$ & 135.0457 (100), 134.03809 (42), $79.0562(6)$ \\
\hline 7 & Ferulic acid & 1.60 & 193.05 & $\mathrm{C}_{10} \mathrm{H}_{10} \mathrm{O}_{4}$ & $\begin{array}{c}134.0375 \text { (100), } 179.0275 \text { (50), } 133.0295 \text { (18), } 149.0611 \\
\text { (14) }\end{array}$ \\
\hline 8 & 6-Hydroxyluteolin -7-O-glucuronide & 1.66 & 477.07 & $\mathrm{C}_{21} \mathrm{H}_{18} \mathrm{O}_{13}$ & $301.0346(100)$ \\
\hline 9 & 6-Hydroxyluteolin-7-O-glucoside & 1.93 & 463.09 & $\mathrm{C}_{21} \mathrm{H}_{20} \mathrm{O}_{12}$ & $301.0331(15)$ \\
\hline 10 & Scutellarin & 2.16 & 461.07 & $\mathrm{C}_{21} \mathrm{H}_{18} \mathrm{O}_{12}$ & 286.0417 (100), 285.0388 (75), 113.0228 (10), $175.0240(5)$ \\
\hline 11 & Narirutin & 2.20 & 579.18 & $\mathrm{C}_{27} \mathrm{H}_{32} \mathrm{O}_{14}$ & 271.0603 (79), $151.0038(9)$ \\
\hline 12 & Rosmarinic acid-3-O-glucoside & 2.22 & 521.13 & $\mathrm{C}_{24} \mathrm{H}_{26} \mathrm{O}_{13}$ & 359.0759 (100), 341.0851 (54) \\
\hline 13 & Luteolin-7-O-glucoside & 2.23 & 447.09 & $\mathrm{C}_{21} \mathrm{H}_{20} \mathrm{O}_{11}$ & $285.0382(10)$ \\
\hline 14 & Isorhamnetin-3-O-rutinoside & 2.24 & 623.16 & $\mathrm{C}_{28} \mathrm{H}_{32} \mathrm{O}_{16}$ & $315.0740(48)$ \\
\hline 15 & Salvianolic acid B & 2.25 & 717.14 & $\mathrm{C}_{36} \mathrm{H}_{30} \mathrm{O}_{16}$ & $519.0863(100)$ \\
\hline 16 & Isorhamnetin-3-O-glucoside & 2.28 & 477.10 & $\mathrm{C}_{22} \mathrm{H}_{22} \mathrm{O}_{12}$ & $315.0740(22)$ \\
\hline 17 & Rosmarinic acid & 2.46 & 359.07 & $\mathrm{C}_{18} \mathrm{H}_{16} \mathrm{O}_{8}$ & $\begin{array}{c}161.0225 \text { (100), } 179.0322 \text { (77), } 197.0425 \text { (63), } 133.0281 \\
\text { (57) }\end{array}$ \\
\hline 18 & Sagerinic acid & 2.46 & 719.16 & $\mathrm{C}_{36} \mathrm{H}_{32} \mathrm{O}_{16}$ & $359.0735(100), 179.0337(7)$ \\
\hline 19 & Luteolin-7-O-glucuronide & 2.57 & 461.07 & $\mathrm{C}_{21} \mathrm{H}_{18} \mathrm{O}_{12}$ & $285.0385(100)$ \\
\hline 20 & Apigenin-7-O-glucoside & 2.58 & 431.10 & $\mathrm{C}_{21} \mathrm{H}_{20} \mathrm{O}_{10}$ & $269.0445(35)$ \\
\hline 21 & Hispidulin glucuronide & 2.65 & 475.09 & $\mathrm{C}_{22} \mathrm{H}_{20} \mathrm{O}_{12}$ & $299.0546(34)$ \\
\hline 22 & Hesperidin & 2.65 & 609.18 & $\mathrm{C}_{28} \mathrm{H}_{34} \mathrm{O}_{15}$ & $301.0697(100)$ \\
\hline 23 & Luteolin & 2.88 & 285.04 & $\mathrm{C}_{15} \mathrm{H}_{10} \mathrm{O}_{6}$ & 133.0300 (24), 151.0042 (12), 175.0409 (9), 199.0406 (9) \\
\hline 24 & Methyl rosmarinate & 2.94 & 373.09 & $\mathrm{C}_{19} \mathrm{H}_{18} \mathrm{O}_{8}$ & $\begin{array}{c}175.0407 \text { (100), } 179.0356 \text { (58), } 197.0463 \text { (45), } 135.0458 \\
\text { (35) }\end{array}$ \\
\hline 25 & Luteolin 3'-acetyl-O-glucuronide & 3.07 & 503.08 & $\mathrm{C}_{23} \mathrm{H}_{20} \mathrm{O}_{13}$ & 443.0579 (100), 285.7495 (9) \\
\hline 26 & Apigenin & 3.47 & 269.04 & $\mathrm{C}_{15} \mathrm{H}_{10} \mathrm{O}_{5}$ & 117.0363 (18), 151.0053 (13), 225.0583 (8) \\
\hline 27 & Hesperetin & 3.48 & 301.07 & $\mathrm{C}_{16} \mathrm{H}_{14} \mathrm{O}_{6}$ & 284.0319 (100), $136.9878(20), 164.0113(5)$ \\
\hline 28 & Diosmetin & 3.56 & 299.05 & $\mathrm{C}_{16} \mathrm{H}_{12} \mathrm{O}_{6}$ & 284.0331 (100), 136.9876 (20), 227.0346 (11) \\
\hline 29 & Epirosmanol & 3.97 & 345.17 & $\mathrm{C}_{20} \mathrm{H}_{26} \mathrm{O}_{5}$ & 284.1679 (12), 283.8697 (4) \\
\hline 30 & Pectolinarigenin & 4.07 & 313.07 & $\mathrm{C}_{17} \mathrm{H}_{14} \mathrm{O}_{6}$ & 298.0471 (100), $283.0241(73), 255.0289(28), 117.0350(6)$ \\
\hline 31 & Rosmanol & 4.18 & 345.17 & $\mathrm{C}_{20} \mathrm{H}_{26} \mathrm{O}_{5}$ & $301.1803(100), 284.0282(6)$ \\
\hline 32 & Rosmadial isomer & 4.42 & 343.15 & $\mathrm{C}_{20} \mathrm{H}_{24} \mathrm{O}_{5}$ & $284.1418(12), 299.8915(7)$ \\
\hline 33 & Epiisorosmanol & 4.46 & 345.17 & $\mathrm{C}_{20} \mathrm{H}_{26} \mathrm{O}_{5}$ & $284.1701(22), 283.8717(8)$ \\
\hline 34 & Genkwanin & 4.46 & 283.06 & $\mathrm{C}_{16} \mathrm{H}_{12} \mathrm{O}_{5}$ & 268.0378 (94), 240.0433 (13), 117.0354 (6), 211.0405 (5) \\
\hline 35 & Rosmadial & 4.60 & 343.15 & $\mathrm{C}_{20} \mathrm{H}_{24} \mathrm{O}_{5}$ & $299.8914(8), 285.1857(6)$ \\
\hline 36 & Rosmanol isomer & 4.97 & 345.17 & $\mathrm{C}_{20} \mathrm{H}_{26} \mathrm{O}_{5}$ & 301.1803 (100), $263.2028(6)$ \\
\hline 37 & Asiatic acid & 5.24 & 487.35 & $\mathrm{C}_{30} \mathrm{H}_{48} \mathrm{O}_{5}$ & - \\
\hline 38 & Rosmanol methyl ether & 5.38 & 359.18 & $\mathrm{C}_{21} \mathrm{H}_{28} \mathrm{O}_{5}$ & 283.8754 (100), 329.1709 (10), 300.1700(15) \\
\hline 39 & Carnosol & 5.63 & 329.17 & $\mathrm{C}_{20} \mathrm{H}_{26} \mathrm{O}_{4}$ & 285.1831 (100), 201.0909 (4), 270.1615 (3), 214.1001 (3) \\
\hline 40 & Carnosic acid & 5.63 & 331.19 & $\mathrm{C}_{20} \mathrm{H}_{28} \mathrm{O}_{4}$ & 286.1848 (100), $287.0588(72)$ \\
\hline 41 & Rosmadial isomer & 5.90 & 343.15 & $\mathrm{C}_{20} \mathrm{H}_{24} \mathrm{O}_{5}$ & 299.2005 (100), 284.1779 (67), 269.1556 (9) \\
\hline 42 & Unknown compound & 6.17 & 469.34 & $\mathrm{C}_{30} \mathrm{H}_{46} \mathrm{O}_{4}$ & $425.3430(10)$ \\
\hline 43 & Corosolic acid & 6.48 & 471.35 & $\mathrm{C}_{30} \mathrm{H}_{48} \mathrm{O}_{4}$ & - \\
\hline 44 & 12-Methoxy-carnosic acid & 6.85 & 345.20 & $\mathrm{C}_{21} \mathrm{H}_{30} \mathrm{O}_{4}$ & 301.2161 (100), 287.1956 (39) \\
\hline 45 & Micromeric acid & 7.53 & 453.33 & $\mathrm{C}_{30} \mathrm{H}_{46} \mathrm{O}_{3}$ & - \\
\hline 46 & Betulinic acid & 7.80 & 455.35 & $\mathrm{C}_{30} \mathrm{H}_{48} \mathrm{O}_{3}$ & - \\
\hline 47 & Ursolic acid & 7.94 & 455.35 & $\mathrm{C}_{30} \mathrm{H}_{48} \mathrm{O}_{3}$ & - \\
\hline
\end{tabular}




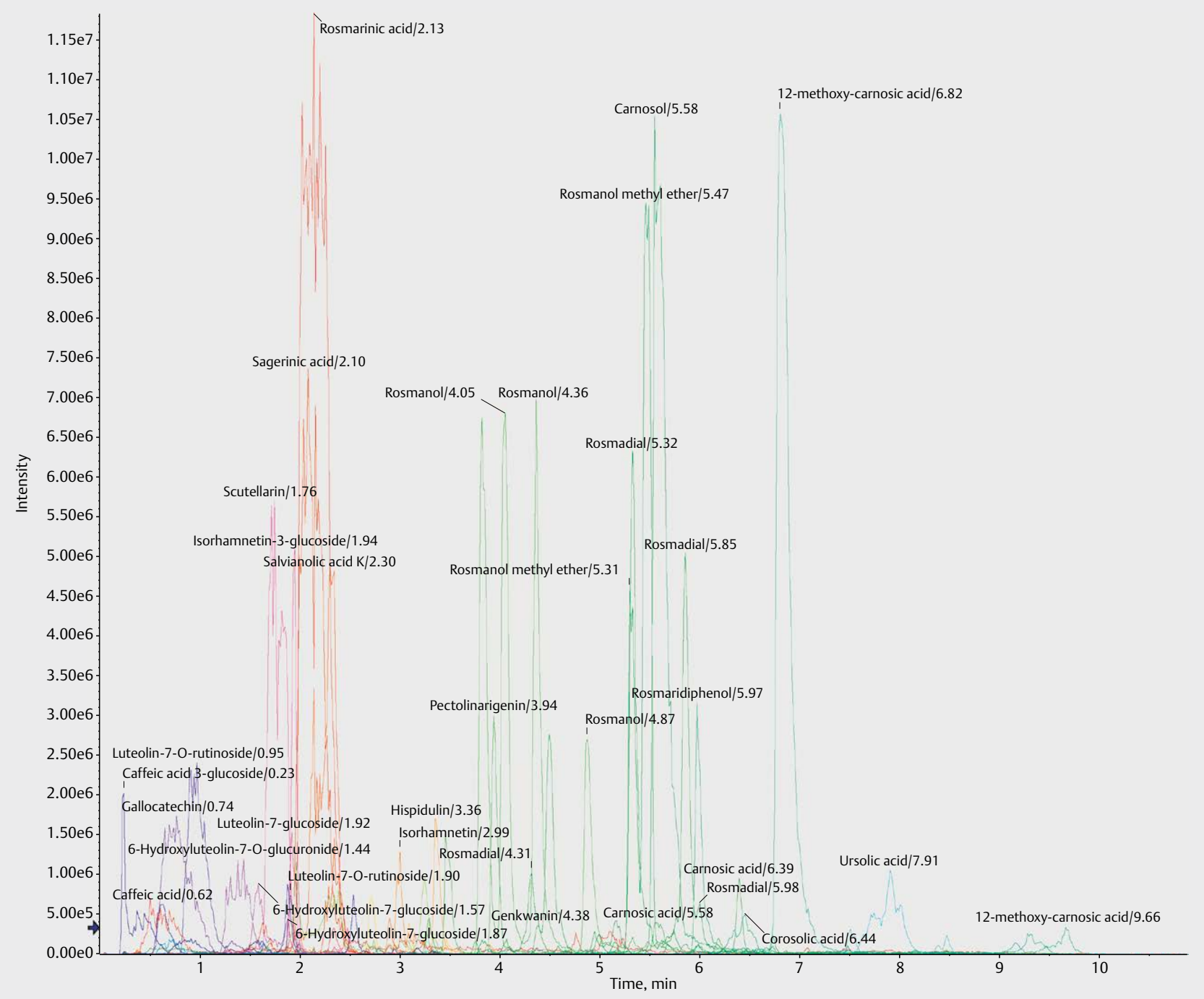

Fig. 2 Chromatogram representing relative abundance of polyphenols in sage leaves analyzed through UHPLC-ESI-QTOF-MS (intensity vs. elution time).

formed by esterification of caffeic acid [42]. Similarly, sagerinic acid $\left([\mathrm{M}-\mathrm{H}]^{-} \mathrm{m} / \mathrm{z} 719.16\right)$ shares an MS/MS ion fragment (359.07) of rosmarinic acid ([M - H] $\mathrm{m} / \mathrm{z} 359.08)$, as has been reported previously by Lu and Foo [43].

A large group of phenolic terpenoid compounds was detected in both sage and rosemary leaves. Rosmanol, rosmadial, and their isomers, carnosol, carnosic acid, rosmanol methyl ether, and 12-methoxy carnosic acids were the major diterpenoids found in the extracts. The presence of more than one peak corresponding to the same molecular mass but with different elution times was due to the presence of isomers. Rosmanol ([M - H] $\left.{ }^{-} \mathrm{m} / \mathrm{z} 345.17\right)$ generated four peaks at different intervals with the same fragmentation (MS² $\mathrm{m} / z$ fragment 284.1 and 301.18 ) ( $\vee$ Figs. 2 and $>\mathbf{3}$ ). Similarly, three peaks were observed for the rosmadial molecule $\left([\mathrm{M}-\mathrm{H}]^{-} \mathrm{m} / \mathrm{z} 343.15\right)$, sharing the same fragmentation patterns. Elution of multiple peaks with the same molecular mass and fragment ions at different retention times indicates the presence of isomers of the same molecules, and similar results were reported in sage and rosemary in previous literature $[28,44,45]$. A total of five pentacyclic triterpenoid compounds, asiatic acid $\left([\mathrm{M}-\mathrm{H}]^{-} \mathrm{m} / \mathrm{z}\right.$ 487.34), betulinic acid and ursolic acid ([M- $\left.\mathrm{H}]^{-} \mathrm{m} / \mathrm{z} 455.35\right)$, corosolic acid ([M - H $]^{-} \mathrm{m} / \mathrm{z} 471.34$, and micromeric acid ([M-H] ${ }^{-} \mathrm{m} / \mathrm{z}$ 453.33), have been identified both in sage and rosemary. Among them, betulinic acid and ursolic acid were detected before in sage and rosemary leaves $[12,26,27,46,47]$. Despite the similar molecular weight of ursolic acid and betulinic acid ([M - H] ${ }^{-}$at $\mathrm{m} / \mathrm{z}$ 455.35), the former was identified through the reference standard, and the later molecule was confirmed by comparison to the NIST mass spectral library. Betulinic acid in the herbs were found to have potent antiviral activity against severe acute respiratory syndrome coronavirus [48]. Although asiatic acid was previously reported in rosemary, it was not reported in the previous literature of $S$. officinalis[26]. A triterpenoid compound, corosolic acid ([M - H] ${ }^{-} \mathrm{m} / \mathrm{z}$ 471.34), was detected in both herbs for the first time, yet corosolic acid was identified before in roots of some Salvia species $[49,50]$. Even though micromeric acid ([M - H] $]^{-} \mathrm{m} / \mathrm{z} 453.33$ ) has been re- 


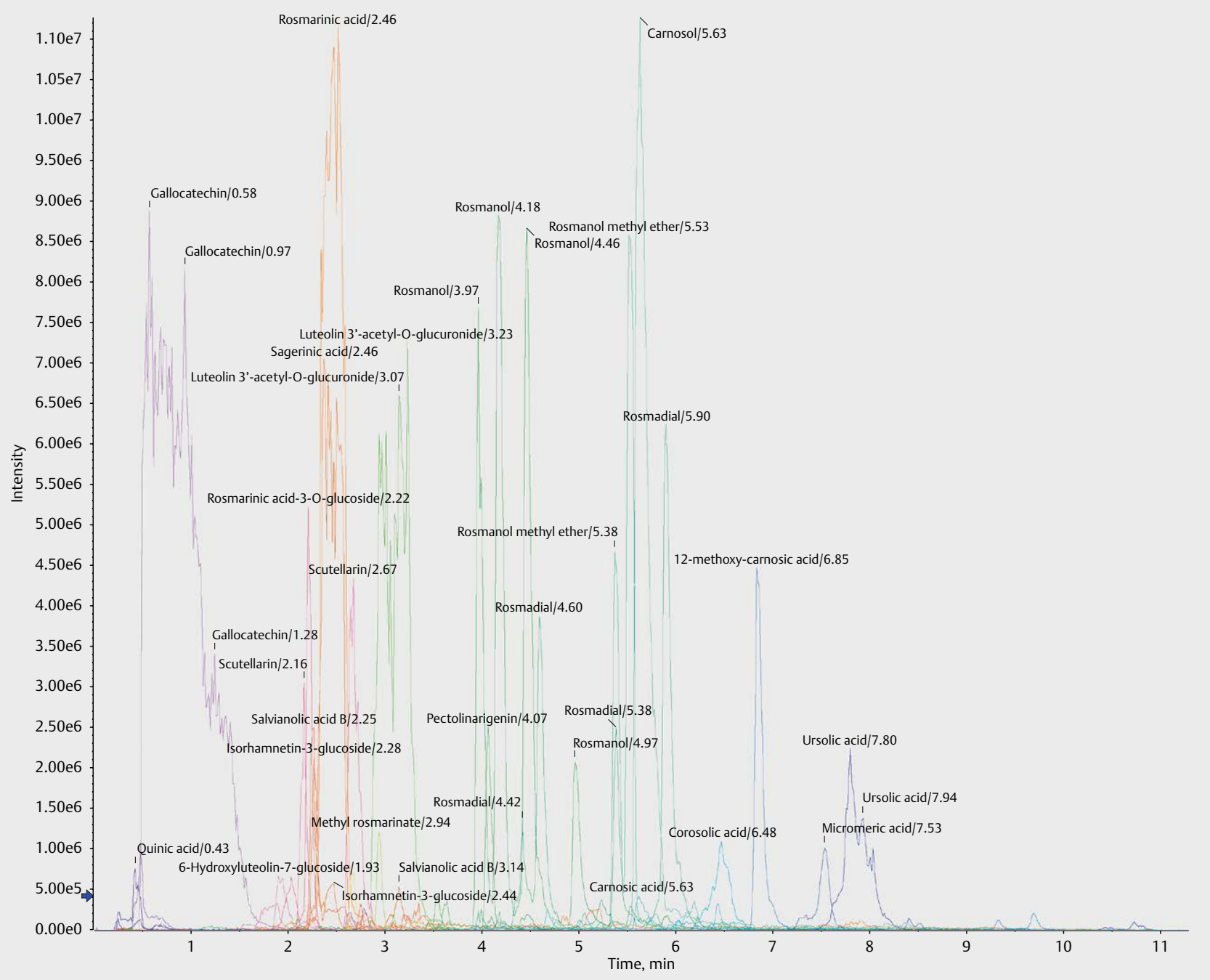

-Fig. 3 Chromatogram representing relative abundance of polyphenols in rosemary leaves analyzed through UHPLC-ESI-QTOF-MS (intensity vs. elution time).

ported in rosemary, it was found for the first time in sage leaves [26]. Triterpene content in rosemary samples was remarkably high compared to sage leaves. Pentacyclic triterpenoids have been reported as having several medicinal properties, especially anti-inflammatory, anticancer, and antidiabetic potential [51, 52].

This study developed a high-throughput, sensitive, and reproducible UHPLC-QTOF-MS/MS method that can serve two purposes. First, it was validated for the identification and quantification of 13 polyphenols in sage and rosemary and could be used for quantitative analysis of any other polyphenol for which reference material is available. Results obtained from validation studies indicated that the developed method was highly selective, sensitive, accurate, and reproducible for the detection and quantitation of target phenolic compounds in complex biological matrices. It has demonstrated great value for qualitative analysis to support the structural elucidation of expected and unexpected metabolites and has been evaluated for its potential to handle quantitative measurements [53]. To the best of our knowledge, this is the most sensitive and comprehensive UHPLC-QTOF-MS/MS-based method for tar- geted analysis of leaf extracts of sage and rosemary. Minor modifications of this method could be applicable as a quantitative tool for accurately and efficiently determining the content of specific phytochemicals present in a wide range of medicinal herbs and extracts thereof given its fast acquisition speed with full-scan sensitivity, enhanced mass resolution, and accurate mass measurement capabilities. Here, we described an optimized and efficient procedure for the UHPLC MS-based analysis of tissue samples from sample preparation to spectral processing and data analysis.

Second, this same UHPLC-QTOF-MS/MS-based method was found to be directly applicable for semiquantitative profiling of the full range of polyphenols in sage and rosemary. The untargeted dimension of this analytical method, enabled by the SWATH protocol, generated retention time, molecular ion $\mathrm{m} / \mathrm{z}$, and mass fragmentation pattern data for virtually every phytochemical present in the sage and rosemary extracts. Many of these phytochemicals were tentatively identified based on the comparison of their molecular $m / z$ and mass fragmentation patterns to data in several mass spectral databases. An equal or larger number of phytochemicals 
- Table 4 Results of analysis of calibration curve and limits of quantification and limits of detection of reference standards.

\begin{tabular}{|c|c|c|c|c|c|c|c|c|c|}
\hline & Standard & $\begin{array}{l}\text { Purity } \\
\text { (\%) }\end{array}$ & Formula & $\begin{array}{l}\text { Molecular } \\
\text { weight }\end{array}$ & $\begin{array}{c}\text { Calibration } \\
\text { range }(\mathrm{ng} / \mathrm{mL})\end{array}$ & LOQ & LOD & Calibration equations & Slope $\left(\mathbf{R}^{2}\right)$ \\
\hline 1 & Apigenin & 100.0 & $\mathrm{C}_{15} \mathrm{H}_{10} \mathrm{O}_{5}$ & 270.24 & $3.91-250$ & 3.81 & 1.65 & $y=0.0311 x+0.01777$ & 0.9926 \\
\hline 2 & Luteolin & 99.6 & $\mathrm{C}_{15} \mathrm{H}_{10} \mathrm{O}_{6}$ & 286.24 & $3.91-250$ & 3.91 & 1.22 & $y=0.04458 x+0.00937$ & 0.9939 \\
\hline 3 & Luteolin-7-O-glucoside & 98.0 & $\mathrm{C}_{21} \mathrm{H}_{20} \mathrm{O}_{11}$ & 448.38 & $3.91-250$ & 3.91 & 1.62 & $y=0.02301 x-0.00426$ & 0.9966 \\
\hline 4 & Hesperidin & 91.1 & $\mathrm{C}_{28} \mathrm{H}_{34} \mathrm{O}_{15}$ & 610.57 & $3.91-250$ & 4.14 & 0.97 & $y=0.01901 x-0.0134$ & 0.9900 \\
\hline 5 & Hesperitin & 99.6 & $\mathrm{C}_{16} \mathrm{H}_{14} \mathrm{O}_{6}$ & 302.28 & $3.91-250$ & 3.70 & 0.88 & $y=0.05678 x+0.04536$ & 0.9907 \\
\hline 6 & Rutin & 96.9 & $\mathrm{C}_{27} \mathrm{H}_{30} \mathrm{O}_{16}$ & 610.56 & $3.91-250$ & 4.14 & 0.96 & $y=0.01645 x-0.02858$ & 0.9892 \\
\hline 7 & Narirutin & 98.6 & $\mathrm{C}_{27} \mathrm{H}_{32} \mathrm{O}_{14}$ & 580.50 & $3.91-250$ & 4.12 & 1.45 & $y=0.01884 x-0.025$ & 0.9906 \\
\hline 8 & Caffeic acid & 98.0 & $\mathrm{C}_{9} \mathrm{H}_{8} \mathrm{O}_{4}$ & 180.00 & $3.91-250$ & 3.91 & 1.87 & $y=0.01048 x+0.01989$ & 0.9964 \\
\hline 9 & Chlorogenic acid & 99.0 & $\mathrm{C}_{16} \mathrm{H}_{18} \mathrm{O}_{9}$ & 354.31 & $3.91-250$ & 3.81 & 1.41 & $y=0.01472 x+0.00617$ & 0.9960 \\
\hline 10 & Rosmarinic acid & 98.0 & $\mathrm{C}_{18} \mathrm{H}_{16} \mathrm{O}_{8}$ & 360.31 & $3.91-250$ & 3.72 & 1.03 & $y=0.02319 x+0.59972$ & 0.9943 \\
\hline 11 & Carnosol & 100.0 & $\mathrm{C}_{20} \mathrm{H}_{26} \mathrm{O}_{4}$ & 330.40 & $3.91-250$ & 3.85 & 1.25 & $y=0.04408 x+0.44018$ & 0.9960 \\
\hline 12 & Carnosic acid & 96.0 & $\mathrm{C}_{20} \mathrm{H}_{28} \mathrm{O}_{4}$ & 332.43 & $15.63-250$ & 14.71 & 6.90 & $y=0.00153 x+0.02517$ & 0.9880 \\
\hline 13 & Ursolic acid & 97.0 & $\mathrm{C}_{30} \mathrm{H}_{48} \mathrm{O}_{3}$ & 456.70 & $3.91-250$ & 4.00 & 1.31 & $y=0.01537 x+0.01792$ & 0.9923 \\
\hline
\end{tabular}

present in the extracts remain unidentified since no compound was identified within the mass spectral databases that corresponded to the compounds detected in the sage and rosemary extracts. These compounds, which numbered 65 in sage and 54 in rosemary, were found in higher intensities with a clear peak area and fragmentation. Most of these compounds were found to be the derivatives or dimers or trimers of luteolin, rosmarinic acid, caffeic acid, rosmanol, rosmadial, and carnosol, matching their fragmentation with their original molecule. However, there is a need to study these unknown molecules using further advanced techniques like NMR spectroscopy for identification and confirmation of these unknown molecules. Thus, the untargeted dimension of this method has contributed to the elucidation of the phytochemical composition of sage and rosemary leaf extracts by defining the mass spectra of the majority of compounds present in these extracts and deducing the tentative identities of many of these by comparison with mass spectral libraries. This sets the stage for additional research identifying the structures and functions of the currently unnamed compounds within these extracts, leading to a complete polyphenol profile of these herbal extracts from which an herbal fingerprint could be developed and used for efficient and accurate routine quality control. It should be noted that the model set forth in this paper should be roughly applicable to create quality control tools for any other herbal material.

A thorough and complete polyphenol profiling of sage and rosemary was made possible by using an ionization technique that was consistently applied to a wide range of molecular structures. We used mass spectral library searching to identify non-targeted compounds based on their mass spectral fragmentation patterns. This strategy of identifying compounds based on their molecular fragmentation fingerprint is very useful and very powerful but is limited to the compounds present in the MS/MS libraries available. This method brought to light a large number of compounds that were not represented in any of the mass spectral libraries available at this time. Thus, they remain unknown in structure and function, although the QTOF analysis did provide a highly accurate molecular mass. These compounds, which numbered 65 in sage and 54 in rosemary, represent an area of great interest for further study. As the research in this field grows, new compounds are added to the databases by the researchers, and the libraries will thus expand. This growing database information will undoubtedly help imminent researchers to effectively use the data to avoid many falsepositive results. This strategy can be further developed into a comprehensive methodology for defining a fingerprint of the queried sample that can be used as a quality control indicator, which can then be used for assessing the quality of sage or rosemary plant material or extracts. This analytical metabolomic strategy of the unknown identification using compound databases will help researchers for putative compound class identification of plant-specific metabolites through non-targeted screening, reducing the economic burden of procuring many stock standards. There is considerable potential for identifying unknown novel compounds in the extracts using this approach.

\section{Materials and Methods}

\section{Chemicals}

LCMS grade methanol and acetonitrile were procured from Honeywell and formic acid and glacial acetic acid were purchased from Merck. Reference standards, apigenin, luteolin, rutin, chlorogenic acid, hesperidin, and hesperetin were obtained from Sigma-Aldrich. Caffeic acid, carnosic acid, luteolin-7-O-glucoside, ursolic acid, and rosmarinic acid were procured from Toronto Research Chemicals. Narirutin and carnosol were from Cayman Chemical. Ultrapure water produced using a Milli-Q A10 water purification system (Millipore Sigma) was used throughout.

\section{Sample collection and extraction}

S. officinalis (voucher no. ISC-454696) and R. officinalis (voucher no. ISC-454695) leaves were collected from the Regenerative Organic Farm, Maharishi University of Management, Fairfield, lowa, USA, during the year 2018. Sage seeds for the experiment were collected from Ohio Heirloom Seeds, and rosemary seeds were collected 
from Everwilde Farms, Sand Creek, WI, USA. Sage seeds were started in the greenhouse in March, and 45-day-old seedlings were transplanted into the main field in the first week of May. Rosemary seedlings were started in November, and 6-week-old seedlings were transplanted into pots and then into the main field in May. Sage leaves were harvested 3 months after planting (in September 2018), and rosemary was harvested 10 months after planting (in October 2018). Freshly harvested leaves were spread over muslin cloth and shade dried for 10 days, then were powdered for extraction and further chromatographic analysis. Next, $5 \mathrm{~g}$ of dry leaf powder were extracted ultrasonically in $50 \mathrm{~mL}$ LCMS grade methanol for $1 \mathrm{~h} 30 \mathrm{~min}$ with a frequency of $40 \mathrm{kHz}$ in a Bransonic-52 ultrasonic bath unit from Branson. Finally, $10 \mathrm{~mL}$ of the extract were centrifuged, and $9 \mathrm{~mL}$ of supernatant were lyophilized and stored in the dark at $-20^{\circ} \mathrm{C}$ until analysis.

\section{UHPLC-QTOF- MS/MS conditions}

Chromatographic analysis was performed on a Shimadzu Nexera X2 UHPLC system (Shimadzu Corporation) equipped with an LC30AD binary pump, SIL-30AC autosampler, and a CTO-30A column oven. The column was a Kinetex XB C18 $(1 \mathrm{~mm} \times 50 \mathrm{~mm}, 2.6 \mathrm{~m}$, $100 \AA$ pore size; Phenomenex) with a KrudKatcher UHPLC in-line filter $(0.5 \mu \mathrm{m})$, maintained at $45^{\circ} \mathrm{C}$. A mixture of MilliQ water $(A)$ and $0.1 \%$ acetic acid in acetonitrile (B) was used as a mobile phase with a flow rate of $0.25 \mathrm{~mL} / \mathrm{min}$ using a multi-step linear gradient elution: 0 min, $10 \% \mathrm{~B} ; 10 \mathrm{~min}, 90 \% \mathrm{~B} ; 12.5 \mathrm{~min}, 90 \% \mathrm{~B} ; 15 \mathrm{~min}, 10 \%$ B; 20 min, $10 \%$ B. The total run time was 20 min, including time for re-equilibration to initial conditions. The sample autosampler injection volume was $10 \mu \mathrm{L}$.

Mass spectrometry was performed using a Triple TOF-5600 (AB SCIEX), a hybrid triple QTOF mass spectrometer equipped with an ESI source, with the mass range set at $m / z 50-1000$. ESI was set in the negative mode with an ion spray needle voltage set at $4500 \mathrm{~V}$ and ion source gas- 1 and gas- 2 each set at 50 psi. The curtain gas was 35 psi and the collision gas pressure was 20 psi with a source temperature of $600{ }^{\circ} \mathrm{C}$. The declustering potential was $-50 \mathrm{eV}$ in TOF-MS and MS/MS experiments, whereas the collision energy values for TOF-MS were $5 \mathrm{eV}$, and for MS/MS experiments, $25 \mathrm{eV}$ with a spread of $15 \mathrm{eV}$.

For SWATH-MS2 acquisition in the collision cell (Q2), nitrogen gas was used for the fragmentation of the precursor ions. In the SWATH-MS ${ }^{2}$ acquisition, a variable SWATH window was used to cover the mass range of $\mathrm{m} / \mathrm{z} 50-1000$ in 16 segments $(15 \times 48.5$ $\mathrm{msec}$ ), yielding a cycle time of $0.8268 \mathrm{sec}$, which included one 50 msec TOF-MS scan. A variable window of the SWATH-MS² generates complex MS/MS spectra that are a composite of the spectra for all of the analytes that elute in that window.

\section{Sample analysis}

Sage and rosemary leaf extracts were dissolved in $4.5 \mathrm{~mL}$ of $10 \%$ acetonitrile and $0.1 \%$ acetic acid. Samples were further diluted in $10 \%$ acetonitrile ( $1 / 200$ to $1 / 40000)$ to bring the levels of analytes within the linear range of the standard calibration curve, thereby avoiding MS signal saturation. Of diluted sample, $190 \mu \mathrm{L}$ were transferred to an autosampler vial along with $10 \mu \mathrm{L}$ of internal standard (chloramphenicol) before analysis. Chloramphenicol was used to correct the instrument variability between injections, normalize the signal intensities, and check the instruments' response over the analytical run. Chloramphenicol, a small molecule, ionizes well in the negative mode of ionization and gives good sensitivity due to the presence of terminal hydroxyl groups. With the ESI interface, like the majority of polyphenols, chloramphenicol forms single charged ions $[\mathrm{M}-\mathrm{H}]^{-}$at $\mathrm{m} / \mathrm{z} 321.0154$. Chloramphenicol chromatography was well studied in our lab and found to be suitable for any $\mathrm{C} 18$ column with a generic gradient with long run times. The standard calibration curves for apigenin, luteolin, luteolin-7-O-glucoside, hesperidin, hesperetin, rutin, narirutin, caffeic acid, chlorogenic acid, rosmarinic acid, carnosol, carnosic acid, and ursolic acid were constructed for quantification of those compounds using chloramphenicol as the internal standard. - Table 4 represents calibration parameters, including LOQ, LOD, calibration range, regression equations, and slopes. The sensitivity, accuracy, and precision of the method were also determined. The mobile phase constituents and the chromatographic gradient were adopted from Gifford et al. with slight modifications, which, together with optimized mass spectrometric conditions, maximized sensitivity and linearity [54]. All extractions and analyses were made in six replications, and the results of polyphenol quantification are expressed as the mean \pm standard deviation.

Unknown polyphenolic compounds and flavonoids were identified based on their accurate mass $(\mathrm{m} / \mathrm{z})$ and molecular $(\mathrm{m} / \mathrm{z})$ ion fragmentation pattern using Peak View Software, ver.2.2, Master View, Library View (all from AB SCIEX), and several mass spectral libraries such as NIST - 2017, AOI (All-in-One) HR-MS/MS Spectral Library 2.0 (Sciex), MassBank of North America (MoNA), and HILIC library database from University of California, Davis.

\section{Supporting Information}

Preparation of standard cocktail tables and fragments of standard compounds are presented in the Supporting Information.

\section{Funding}

Special thanks to Health Research Institute, Fairfield, lowa for funding and providing the facility to analyze the samples

Conflict of Interest

The authors declare that they have no conflict of interest.

References

[1] Andrews GL, Simons BL, Young B], Hawkridge AM, Muddiman DC. Performance characteristics of a new hybrid triple quadrupole time-of-flight tandem mass spectrometer. Anal Chem 2011; 83: $5442-5446$

[2] Zhu X, Chen Y, Subramanian R. Comparison of information-dependent acquisition, SWATH, and MSAll techniques in metabolite identification study employing ultrahigh-performance liquid chromatography- quadrupole time of-flight mass spectrometry. Anal Chem 2014; 86: $1202-1209$ 
[3] Kaufmann A. Combining UHPLC and high-resolution MS: A viable approach for the analysis of complex samples? Trends in Analytical Chemistry 2014; 63: 113-128

[4] Li H, Yao W, Liu Q, Xu J, Bao B, Shan M, Cao Y, Cheng F, Ding A, Zang L. Application of UHPLC-ESI-Q-TOF-MS to identify multiple constituents in processed products of the herbal medicine Ligustri Lucidi Fructus. Molecules 2017; 22: 689

[5] Kumar S, Singh A, Kumar B. Identification and characterization of phenolics and terpenoids from ethanolic extracts of Phyllanthus species by HPLC-ESI-QTOF-MS/MS. J Pharm Anal 2017; 2: 214-222

[6] Jin Y, Ma Y, Xie W, Hou L, Xu H, Zhang K, Zhang L, Du Y. UHPLC-Q-TOFMS/MS-oriented characteristic components dataset and multivariate statistical techniques for the holistic quality control of Usnea. RAC Adv 2018; 8: 15487-15500

[7] Brighenti V, Groothuisa SF, Prencipe PF, Amir R, Benvenuti F, Pellati F. Metabolite fingerprinting of Punica granatum L. (pomegranate) polyphenols by means of high-performance liquid chromatography with diode array and electrospray ionization-mass spectrometry detection. J Chromatogr A 2017; 1480: 20-31

[8] Tzima K, Brunton NP, Rai DK. Qualitative and quantitative analysis of polyphenols in lamiaceae. plants-A review. Plants 2018; 7: 25

[9] Cajka T, Fiehn O. Comprehensive analysis of lipids in biological systems by liquid chromatography-mass spectrometry. Trends Analyt Chem 2014; 61: 192-206

[10] Genena AK, Hense H, Smânia Junior A, Machado de Souza S. Rosemary (Rosmarinus officinalis): A study of the composition, antioxidant and antimicrobial activities of extracts obtained with supercritical carbon dioxide. Ciência e Tecnol Aliment 2008; 28: 463-469

[11] Hamidpour M, Hamidpour R, Hamidpour S, Shahlari M. Chemistry, Pharmacology and Medicinal Property of Sage (Salvia) to Prevent and Cure Illnesses such as Obesity, Diabetes, Depression, Dementia, Lupus, Autism, Heart Disease and Cancer. J Tradit Complement Med 2014; 4: $82-88$

[12] Ghorbani A, Esmaeilizadeh M. Pharmacological properties of Salvia officinalis and its components. J Tradit Complement Med 2017; 7: 433-440

[13] Garcia CSC, Menti C, Lambert APF. Pharmacological perspectives from Brazilian Salvia officinalis (Lamiaceae): Antioxidant, and antitumor in mammalian cells. An Acad Bras de Cienc 2016; 88: 281-292

[14] Lopresti AL. Salvia (Sage): A Review of its Potential Cognitive-Enhancing and Protective Effects. Drugs R D 2017; 17: 53-64

[15] Sharma Y, Fagan J, Schaefer J. Ethnobotany, phytochemistry, cultivation and medicinal properties of Garden sage (Salvia officinalis L.). J Pharmacogn Phytochem 2019; 8: 3139-3148

[16] Miraj S, Kiani S. A review study of therapeutic effects of Salvia officinalis L. Der Pharm Lett 2016; 8: 299-303

[17] Hamidpour R. Medicinal Property of Sage (Saliva) for Curing Illnesses Such as Obesity, Diabetes, Depression, Dementia, Lupus, Autism, Heart Disease and Cancer: A Brief Review. Arch. Cancer Res 2015; 3: $41-44$

[18] Walch S, Tinzoh L, Zimmerman B, Stuhlinger W, Lachenmeier D. Antioxidant Capacity and Polyphenolic Composition as Quality Indicators for Aqueous Infusions of Salvia officinalis L. (sage tea). Front Pharmacol 2011; 2: 79

[19] Minaiyan M, Ghannadi AR, Afsharipour M, Mahzouni P. Effects of extract and essential oil of Rosmarinus officinalis L. on TNBS-induced colitis in rats. Res Pharm Sci 2011; 6: 13-21

[20] Orhan I, Aslan S, Kartal M, Şener B, Baser KHC. Inhibitory effect of Turkish Rosmarinus officinalis L. on acetylcholinesterase and butyrylcholinesterase enzymes. Food Chem 2008; 108: 663-668

[21] Al Sereitia MR, Abu-Amerb KM, Sena P. Pharmacology of rosemary (Rosmarinus officinalis Linn.) and its therapeutic potentials. Indian J Exp Biol 1999; 37: 124-131
[22] Jordan M], Lax V, Rota MC, Loran S, Sotomayor JA. Effect of bioclimatic area on the essential oil composition and antibacterial activity of Rosmarinus officinalis L. Food Control 2013; 30: 463-468

[23] Kontogianni VG, Tomic G, Nikolic I, Nerantzaki AA, Sayyad N, Stosic-Grujicic S, Stojanovic I, Gerothanassis IP, Tzakos AG. Phytochemical profile of Rosmarinus officinalis and Salvia officinalis extracts and correlation to their antioxidant and anti-proliferative activity. Food Chem 2013; 136: 120-129

[24] Lu Y, Foo LY. Polyphenolics of Salvia-a review. Phytochem 2002; 59 : $117-140$

[25] Neagu E, Paun G, Radu GL. Chemical composition and antioxidant activity of Salvia officinalis concentrated by ultrafiltration. Rom Biotechnol Lett 2014; 19: 9203-9211

[26] Borras-Linares I, Stojanovic Z, Quirantes-Pine R, Arráez-Román D, Švarc-Gajić j, Fernández-Gutiérrez A, Segura-Carretero A. Rosmarinus officinalis leaves as a natural source of bioactive compounds. Int J Mol Sci 2014; 15: 20585-20606

[27] Mena P, Cirlini M, Tassotti M, Herrlinger KA, Dall’Asta C, DelRio D. Phytochemical profiling of flavonoids, phenolic acids, terpenoids, and volatile fraction of a rosemary (Rosmarinus officinalis L.) extract. Molecules 2016; 21: 1576

[28] Zimmermann BF, Walch SG, Tinzoh LN, Stühlinger W, Lachenmeier DW. Rapid UHPLC determination of polyphenols in aqueous infusions of Salvia officinalis L. (sage tea). J Chromatogr B 2011; 879: 24592464

[29] Jensen PK, Wujcik CE, McGuire MK, McGuire MA. Validation of reliable and selective methods for direct determination of glyphosate and aminomethylphosphonic acid in milk and urine using LC-MS/MS. J Environ Sci Health B 2016; 51: 254-259

[30] Hamrouni-Sellami I, Rahali FZ, Rebey IB, Bourgou S, Limam F, Marzouk B. Total phenolics, flavonoids, and antioxidant activity of Sage (Salvia officinalis L.) plants as affected by different drying methods. Food Bioprocess Technol 2013; 6: 806-817

[31] Martins N, Barros L, Santos-Buelga C, Henriques M, Silva S, Ferreira ICFR. Evaluation of bioactive properties and phenolic compounds in different extracts prepared from Salvia officinalis L. Food Chem 2015; 170: $378-385$

[32] Madala NE, Piater L, Dubery I, Steenkamp P. Distribution patterns of flavonoids from three Momordica species by ultra-high performance liquid chromatography quadrupole time of flight mass spectrometry: A metabolomics profiling approach. Rev Bras Farmacogn 2016; 26: 507-513

[33] Mercolini L, Protti M, Saracino MA, Mandrone M, Antognoni F. Analytical profiling of bioactive phenolic compounds in argan (Argania spinosa) leaves by combined microextraction by packed sorbent (MEPS) and LC-DAD-MS/MS. Phytochem Anal 2016; 27: 41-49

[34] Neumann S, Bocker S. Computational mass spectrometry for metabolomics: identification of metabolites and small molecules. Anal Bioanal Chem 2010; 398: 2779-2788

[35] Cajka T, Fiehn O. Toward merging untargeted and targeted methods in mass spectrometry-based metabolomics and lipidomics. Anal Chem 2015; 88: 524-545

[36] Dunn WB, Erban A, Weber RJM, Creek DJ, Brown M, Breitling R, Hankemeier T, Goodacre R, Neumann S, Kopka J, Viant MR. Mass appeal: Metabolite identification in mass spectrometry-focused untargeted metabolomics. Metabolomics 2012; 9: 44-66

[37] Lee B, Lim C, Lim S, Cho S. Baicalin administered orally after ischemia/ reperfusion alleviated brain injury in mice by inhibiting inflammation and edema. Nat Prod Commun 2019; 14: $1934578 \times 19843032$

[38] Sowndhararajan K, Deepa P, Kim M, Park JS, Kim S. Neuroprotective and cognitive enhancement potentials of Baicalin: A review. Brain Sci 2018; 8: 104 
[39] Borras-Linares I, Arraez-Roman D, Herrero M, Ibanez E, Segura-Carretero A, Fernandez-Gutierrez A. Comparison of different extraction procedures for the comprehensive characterization of bioactive phenolic compounds in Rosmarinus officinalis by reversed-phase high-performance liquid chromatography with diode array detection coupled to electrospray time-of-flight mass spectrometry. J Chromatogr A 2011; 1218: 7682-7690

[40] Lim H, Son KH, Chang HW, Bae K, Kang SS, Kim HP. Anti-inflammatory activity of pectolinarigenin and pectolinarin isolated from Cirsium chanroenicum. Biol Pharm Bull 2008; 31: 2063-2067

[41] Patel K, Gadewar M, Tahilyani V, Patel DK. A review on pharmacological and analytical aspects of diosmetin: a concise report. Chin J Integr Med 2013; 19: 792-800

[42] Wang J, Xu J, Gong X, Yang M, Zhang C, Li M. Antioxidative phenolic compounds from sage (Salvia officinalis). J Agric Food Chem 1998; 46: 4869-4873

[43] Lu Y, Foo LY. Rosmarinic acid derivatives from Salvia officinalis. Phytochem 1999; 51: 91-94

[44] Cuvelier M, Richard H, Berset C. Antioxidative activity and phenolic composition of pilot-plant and commercial extracts of sage and rosemary. JAOCS 1996; 73: 645-652

[45] Hossain MB, Rai DK, Brunton NP, Martin-Diana AB, Barry-Ryan AC. Characterization of phenolic composition in Lamiaceae spices by LC-ESI-MS/MS. J Agric Food Chem 2010; 58: 10576-10581

[46] Jakovljevic M, Jokic S, Molnar M, Jasic M, Babic J, Jukic H, Banjari I. Bioactive Profile of Various Salvia officinalis L. Preparations. Plants 2019; 8: 55

[47] Abdollahi-Ghehi H, Sonboli A, Ebrahimi SN, Esmaeili MA, Mirjalili MH. Triterpenic Acid Content and Cytotoxicity of Some Salvia Species from Iran. Nat Prod Commun 2019; 14: 1934578x19842722
[48] Wen CC, Kuo YH, Jan JT, Linag PH, Wang SY, Liu HG, Lee CK, Chang ST, Kuo C], Lee SS, Hou CC, Hsiao PW, Chien SC, Shyur LF, Yang NS. Specific plant terpenoids and lignoids possess potent antiviral activities against severe acute respiratory syndrome coronavirus. J Med Chem 2007; 50: 4087-4095

[49] Bahadori MB, Dinparast L, Valizadeh H, Farimani MM, Ebrahimi SN. Bioactive constituents from roots of Salvia syriaca L.: Acetylcholinesterase inhibitory activity and molecular docking studies. S Afr J Bot 2016; 106: 1-4

[50] Saeidnia S, Ghamarinia M, Gohari AR, Shakeri A. Terpenes From the Root of Salvia hypoleuca Benth. Daru 2012; 20: 66

[51] Alqahtani A, Hamid K, Kam A, Wong KH, Abdelhak Z, RazmovskiNaumovski V, Chan K, Li KM, Groundwater PW, Li GQ. The pentacyclic triterpenoids in herbal medicines and their pharmacological activities in diabetes and diabetic complications. Curr Med Chem 2013; 20: 908-931

[52] Laszczyk MN. Pentacyclic triterpenes of the lupane, oleanane and ursane group as tools in cancer therapy. Planta Med 2009; 75 : $1549-1560$

[53] Xie C, Zhong D, Yu K, Chen X. Recent advances in metabolite identification and quantitative bioanalysis by LC-Q-TOF MS. Bioanalysis 2012; 4: 937-959

[54] Gifford I, Battenberg K, Vaniya A, Wilson A, Tian L, Fiehn O, Berry AM. Distinctive patterns of flavonoid biosynthesis in roots and nodules of Datisca glomerata and Medicago spp. revealed by metabolomic and gene expression profiles. Front Plant Sci 2018; 9: 1463 